of neutralizing antibody increased (Fig. 1). Antibody effector functions improved, and immune complexes were reduced. Splenic size was normalized, and germinal center sizes and architecture mirrored that seen in typical non-chronic responses. This normalization was a remarkable effect caused by the deletion of Bmi1 - a factor that is usually seen in a positive light in the context of stem cells.

During chronic infections, BMI-1 may be a candidate for therapeutic interventions to improve the quality of the humoral response. However, as mentioned above, BMI- 1 is involved in many essential processes in stem cells and other lineages, including B cell progenitors. Although inhibitors of BMI-1 are moving toward clinical trials for the treatment of cancer, its pleiotropism may be an obstacle to translation for the purposes of improving immune responses. As an alternative, inhibition of upstream cytokines that induce BMI-1 expression may be better therapeutic targets. Interleukin 21 (IL-21) is one such candidate that was shown to induce BMI-1 expression in B cells stimulated in vitro, and for which blocking antibodies are under development for therapeutic purposes.

So is BMI- 1 a villain in all antibody responses? Definitely not. Although systemic small molecule inhibition of BMI-1 in chronically infected mice reduced illness and the levels of poor-quality antibodies, genetic ablation of Bmil also led to a loss of long-lived plasma cells and serum antibodies when animals were immunized with standard hapten-protein conjugates. Therefore, as with all aspects of the immune system, whether a pathway is beneficial or detrimental depends on the context. Often, these contexts depend on pathogens that have learned to exploit the vulnerabilities of the immune system. Thankfully, work by Di Pietro and colleagues helps to explain these vulnerabilities and shows us a path to fight back.

Tyler J. Ripperger and

Deepta Bhattacharya (D) $₫$

Department of Immunobiology, The University of
Arizona College of Medicine, Tucson, AZ, USA.

$凶_{e-m a i l: d e e p t a b @ a r i z o n a . e d u}$

Published online: 20 December 2021

https://doi.org/10.1038/s41590-021-01092-z

References

1. Di Pietro, A. et al. Nat. Immunol. https://doi.org/10.1038/s41590021-01084-z (2021).

2. Ripperger, T. J. \& Bhattacharya, D. Annu. Rev. Immunol. 39, 345-368 (2021).

3. Li, Y. et al. Nat. Med. 13, 1032-1034 (2007).

4. Hunziker, L. et al. Nat. Immunol. 4, 343-349 (2003).

5. Calado, D. P. et al. Nat. Immunol. 13, 1092-1100 (2012).

6. Dominguez-Sola, D. et al. Nat. Immunol. 13, 1083-1091 (2012).

7. Alkema, M. J. et al. Genes Dev. 11, 226-240 (1997).

8. Sangiorgi, E. \& Capecchi, M. R. Nat. Genet. 40, 915-920 (2008).

9. Lessard, J. \& Sauvageau, G. Nature 423, 255-260 (2003).

10. Molofsky, A. V., He, S., Bydon, M., Morrison, S. J. \& Pardal, R. Genes Dev. 19, 1432-1437 (2005).

11. Park, I. et al. Nature 423, 302-305 (2003).

12. Kobayashi, M. et al. J. Immunol. 204, 3262 (2020).

13. Luckey, C. J. et al. Proc. Natl Acad. Sci. USA 103, 3304 (2006).

Competing interests

The authors declare no competing interests.

\title{
Boosted immunity to the common cold might protect children from COVID-19
}

\author{
New data show that, compared with adults, children infected by SARS-CoV-2 preferentially activate pre-existing \\ immunity to endemic common-cold coronaviruses that are cross-reactive with SARS-CoV-2, with potential \\ implications for pediatric vaccine strategies.
}

\section{Jason J. Lavinder and Gregory C. Ippolito}

T he biological age of an individual is the primary risk factor associated with the clinical severity of SARS-CoV-2 infection $^{1,2}$. Although comorbidities (for example, cardiovascular disease and obesity) accumulate with age and thereby increase the chances of severe illness or fatal disease, they cannot explain why increasing age is an independent risk factor, or why, notably, SARS-CoV-2 infection tends to be mild or even asymptomatic in children and young adults. A deeper characterization of these age-related differences could improve our understanding of immune responses to SARS-CoV-2 generally and identify a profile of SARS-CoV-2-specific responses in children following natural infection that might enable optimal vaccination strategies for the young. In this issue of Nature Immunology, Dowell et al. ${ }^{3}$ quantitatively profile the convalescent serological (antibody) and cellular ( $\mathrm{T}$ cell) immune responses to SARS-CoV-2 in a cohort of 91 children and 154 adults - one of the largest cohorts examined to date - who had mild or asymptomatic disease in the United Kingdom during the latter half of 2020. The authors provide evidence that children mount exceptional immune responses, when compared to adults, with pre-existing immunity cross-reactive with pre-pandemic 'common cold' human coronaviruses (HCoVs) as well as SARS-CoV-2. Dowell et al. ${ }^{3}$ postulate that these enhanced immune responses derive from prior $\mathrm{HCoV}$ exposures and demonstrate that they are upregulated, or 'back boosted', by SARS-CoV-2 infection.

The importance of age as a risk factor is underscored by early estimates in 2020 that adults $>65$ years in age represented $80 \%$ of hospitalizations and had a 23 -fold greater risk of death than those $<65$ years of age $^{2}$. This trend has been maintained throughout the pandemic, and according to recent calculations, almost $76 \%$ of $\sim 737,000$ cumulative deaths in the United States (as of 27 October 2021) were of individuals aged $\geq 65$ years $^{4}$. Notably, the hospitalization or death rate in this age range is approximately $1,000-8,000$ times higher than in the 
Young

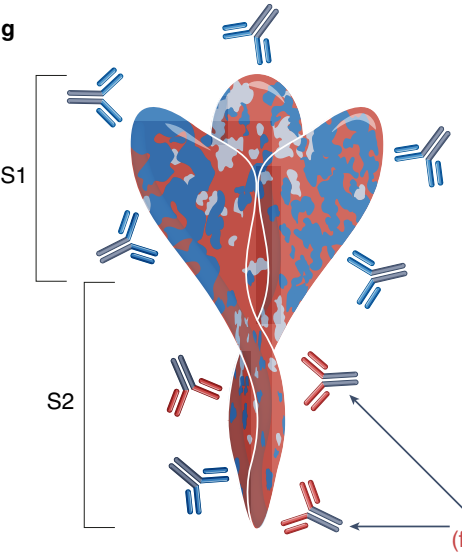

Humoral immunity

Old

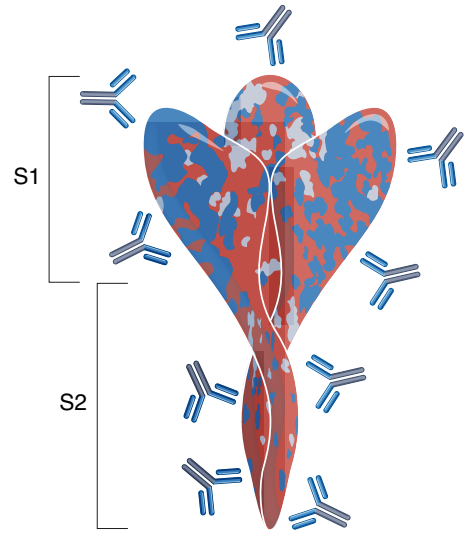

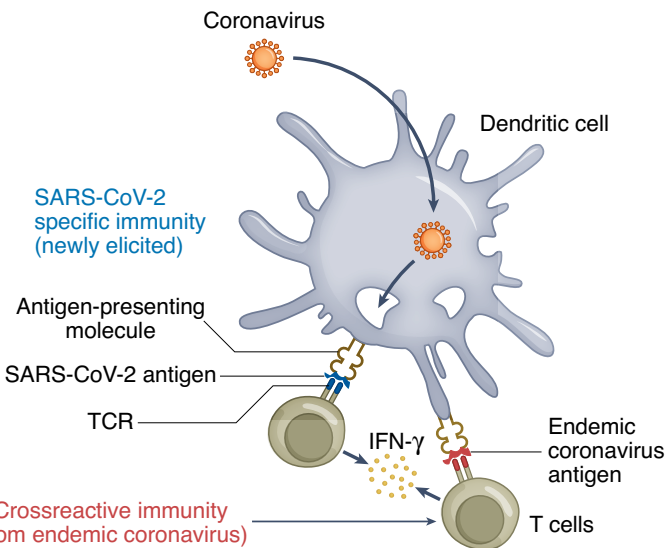

Cellular immunity

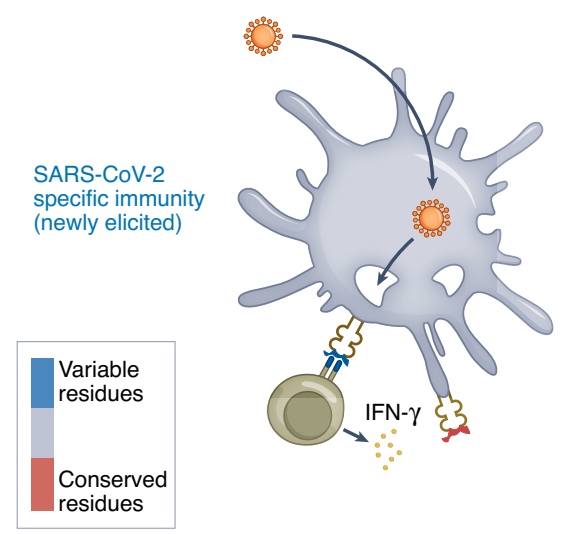

Fig. 1 | Immunity to coronavirus spike glycoprotein in the young and old. The sequence conservation of the spike glycoprotein among alphacoronaviruses and betacoronaviruses is limited in the receptor-binding domain and $\mathrm{N}$-terminal domain of the $\mathrm{S} 1$ subunit of the viral spike protein. However, the SARS-CoV-2 S2 subunit is highly conserved with commonly circulating endemic coronaviruses. As a result, S2-specific immune responses to SARS-CoV-2 are often cross-reactive across the betacoronavirus clade, indicating that some individuals, particularly children who have high levels of immunity to these common viruses, have a pre-existing head start in immunity to SARS-CoV-2 and produce a more robust cross-reactive antibody and T cell response. TCR, T cell antigen receptor.

0-17-year old reference group. In one landmark analysis, the infection fatality ratio was calculated to be the lowest among children (aged 5-9 years), with a log-linear increase by age among individuals older than 30 years ${ }^{1}$. These observations lead us to the question of how immune responses to SARS-CoV-2 might differ in children versus adults.

Although SARS-CoV-2 is a new coronavirus circulating within the human population, the majority of people throughout their lives have been exposed to antigenically related seasonal endemic $\mathrm{HCoVs}$ and have detectable $\mathrm{HCoV}$-specific humoral, B cell and $\mathrm{T}$ cell immune responses ${ }^{5}$. The closely related endemic coronaviruses HKU1 and OC43, in particular, share extensive sequence homology with SARS-CoV-2. Adaptive immune responses to these $\mathrm{HCoVs}$ have been shown to cross-react with SARS-CoV-2 antigens, including the immunodominant spike glycoprotein ${ }^{6-8}$. So, what might be the immunological relationship between pre-existing responses to endemic HCoVs and SARS-CoV-2, and how might such cross-reactivity affect disease outcomes or age-related disparities? One examination of electronic medical records suggested that recent $\mathrm{HCoV}$ infections are associated with a reduction in COVID-19 severity, implying that pre-existing immune responses to endemic $\mathrm{HCoVs}$ might mitigate disease outcomes in SARS-CoV-2 infection"; however, other reports have drawn contrary conclusions ${ }^{10}$.
To address these questions, Dowell et al. assembled a large well-controlled data set of age-matched infected and non-infected children and adults. Using this cohort, the authors could quantitate the recall of cross-reactive antibody and cellular immune responses and how they can differ between the two age groups. All study volunteers had only mild or asymptomatic infections. In children of 3-11 years of age (median 7 years), in comparison to adults $20-71$ years of age (median 41 years), children had statistically distinct immunological profiles of humoral and cellular immunity.

Dowell et al. ${ }^{3}$ first established that SARS-CoV-2-infected children and adults can both produce robust antibody responses (as expected) against the receptor-binding domain, the $\mathrm{N}$-terminal domain and the full ectodomain of the SARS-CoV-2 spike glycoprotein, as measured by an electro-chemiluminescence multiplex assay. Compared with adults, children showed an intriguing yet statistically insignificant trend for overall higher spike-reactive antibody production. With regard to $\mathrm{HCoV}$ antibody levels, titers against $\mathrm{HCoVs}$ were evident in SARS-CoV-2 seropositive children and adults, which were boosted compared to their respective seronegative groups. Antibody levels against all four HCoVs were boosted most in the SARS-CoV-2 seropositive children and reached statistical significance for HKU-1 and OC43. Importantly, no significant change was detected in the antibody titers against unrelated respiratory viruses (that is, influenza A virus and respiratory syncytial virus), demonstrating that this effect was specific to HCoVs. These data indicate that SARS-CoV-2 infection in children specifically and significantly back boosts pre-existing immunity to OC43 and HKU-1. Moreover, the authors demonstrate quantitatively that the back-boosted OC43 and HKU1 plasma antibody in seropositive children is predominantly cross-reactive with the SARS-CoV-2 S2 domain, explaining the higher SARS-CoV-2-specific titer in children.

Dowell et al. ${ }^{3}$ go on to show that the durability of the polyclonal spike-specific humoral immune response in children is robust $\geq 12$ months after infection, and is quantitively superior to antibody responses in adults. For example, compared with adults, children maintain higher levels of antibody binding to the Wuhan SARS-CoV-2 founder virus and to a broader panel of variants of concern (VOC). Although children have better antibody binding to VOC spike proteins, virus-neutralizing capacity is nevertheless equivalent in adults and children. Of note, 
children have superior retention $(>6$ months) of antibodies that bind to non-neutralizing spike epitopes. However, whether these non-neutralizing antibodies provide protection through Fc-mediated functions is unclear. In summary, these data from Dowell and colleagues illustrate how children - after natural infection develop durable titers of polyclonal plasma antibodies that have superior binding to SARS-CoV-2 VOC, but are endowed with similar neutralizing potency when compared with the adult antibody response.

Aside from the humoral immune response, Dowell et al. ${ }^{3}$ compared $\mathrm{T}$ cell responses in children versus adults. Mirroring the antibody profiles, children also developed robust cellular immune responses to the spike protein following SARS-CoV-2 infection. Using an interferon- $\gamma($ IFN- $\gamma$ ) enzyme-linked immune absorbent spot (ELISpot) and overlapping peptide pools derived from the spike glycoprotein and a combination of nucleocapsid, membrane and envelope proteins, $\mathrm{T}$ cell responses were similarly frequent in SARS-CoV-2 seropositive children (33/37) and adults (51/64), but the number of spot-forming cells was twice as high in the children. A reduced number of cross-reactive $T$ cells in adults could result in inadequate control of SARS-CoV-2 infection. Furthermore, using a multi-analyte bead assay and flow cytometry, it was shown that IL-2- $\mathrm{TNF}^{+} \mathrm{IFN}-\gamma^{+} \mathrm{CD}^{+}$ T cells (where IL-2 is interleukin 2; and TNF is tumor necrosis factor) comprise the majority of the spike-specific T cell repertoire, and that IL-2 production is markedly reduced among virus-specific $\mathrm{T}$ cell responses in children. With a combination of SARS-CoV-2 seronegative volunteers and pre-pandemic negative volunteers, the authors have documented the increased presence of $\mathrm{HCoV}-\mathrm{SARS}-\mathrm{CoV}-2$ spike cross-reactive $\mathrm{T}$ cell responses in a high proportion of children.

Although more studies are needed, it is increasingly clear that CD $4^{+} \mathrm{T}$ cells, $\mathrm{CD}^{+} \mathrm{T}$ cells and antibodies (neutralizing and non-neutralizing) all contribute to the collective control of SARS-CoV-2 infection and disease. The continuing study of immunological cross-reactivity between $\mathrm{HCoVs}$ and SARS-CoV-2 and how it might underlie age-related disparity in SARS-CoV-2 clinical outcomes could lead to the rational design of pan-coronavirus therapeutics or vaccines. For example, the data presented here indicate that vaccines designed to induce SARS-CoV-2 spike cross-reactive $\mathrm{T}$ cell immunity and cross-reactive plasma antibodies directed to the S2 domain (in addition to the induction of S1-directed virus-neutralizing antibodies) might provide enhanced protection.

At the bulk serological level, Dowell et al. ${ }^{3}$ have demonstrated that the pool of polyclonal plasma antibodies in children is enriched for the preferential targeting of conserved epitopes shared between $\mathrm{HCoV}$ and SARS-CoV-2 spike glycoproteins (particularly the S2 subunit). This feature indirectly suggests a salutary role for cross-reactive antibodies in the resolution of SARS-CoV-2 viral infection. A general limitation of this study, however, is its exclusive use of pooled polyclonal plasma antibody, not individual monoclonal antibody clones, so it can only be inferred that any given plasma antibody species was either pre-existing or cross-reactive with $\mathrm{HCoVs}$ and SARS-CoV-2. Proteomics methods for the identification and molecular-level quantitation of individual antibodies in polyclonal plasma have been used previously to identify cross-reactive antibody clones within SARS-CoV-2 convalescent plasma, which tend to be S2-directed and frequently constitute $>40 \%$ of all antibodies comprising the polyclonal titer to the spike protein ${ }^{11}$. The role of pre-existing humoral immunity ${ }^{5}$, the cross-reactivity of immune interactions between SARS-CoV-2 and seasonal $\mathrm{HCoVs}^{6,7,9,10}$, the extent to which serological recall and back boosting might be controlled by immune imprinting ${ }^{12}$ and the design of next-generation pan-coronavirus vaccines based on S2 immunogens are all areas of active investigation that should benefit from the data reported by Dowell et al. ${ }^{3}$ (Fig. 1).

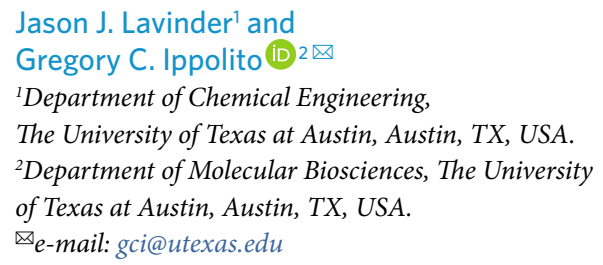

Published online: 22 December 2021

https://doi.org/10.1038/s41590-021-01094-x

References

1. O’Driscoll, M. et al. Nature 590, 140-145 (2021).

2. Mueller, A. L. et al. Aging 12, 9959-9981 (2020).

3. Dowell, A. C. et al. Nat. Immunol. https://doi.org/10.1038/s41590021-01089-8 (2022).

4. Statista https://www.statista.com/statistics/1191568/reporteddeaths-from-covid-by-age-us/ (accessed 27 October 2021).

5. Huang, A. T. et al. Nat. Commun. 11, 4704 (2020).

6. Song, G. et al. Nat. Commun. 12, 2938 (2021).

7. Wec, A. Z. et al. Science 369, 731-736 (2020).

8. Braun, J. et al. Nature 587, 270-274 (2020).

9. Sagar, M. et al. J. Clin. Invest. 4, e143380 (2021).

10. Anderson, E. M. et al. Cell 184, 1858-1864 (2021).

11. Voss, W. N. et al. Science 372, 1108-1112 (2021).

12. Aydillo, T. et al. Nat. Commun. 12, 3781 (2021).

Competing interests

The authors declare no competing interests. 